\title{
EL PRACTICUM I DE PEDAGOGÍA EN LA UNIVERSIDAD NACIONAL DE EDUCACIÓN A DISTANCIA
}

\author{
(THE PRACTICUM AS A SUBJECT OF THE EDUCATION PROGRAM AT THE UNED)
}

Elisa Gavari

Universidad Nacional de Educación a Distancia, UNED (España)

\section{RESUMEN}

En este artículo se explican las peculiaridades de la implantación y desarrollo de la asignatura del Practicum de Pedagogía en la UNED. En particular, se describen las innovaciones que esta materia introduce en la gestión y en la docencia. Respecto de la gestión en el texto se presenta el organigrama con los miembros de la universidad que intervienen y sus funciones. En la parte que se dedica a la docencia se explican los roles del docente y del discente así como los fines y el contenido de la asignatura. Por último, se incluyen unas recomendaciones para los alumnos respecto de las normas básicas para la realización de las prácticas.

\begin{abstract}
This paper shows the continuous efforts to reach high quality in distance education at Unisul Virtual programs. These efforts are concentrated on the role of the teachers, called here Tutors. First of all is showed that this quality is not an isolated factor, but in fact it is part of each process taking advantage of human talents. The tutor role in this scenario is essential for the good development of distance education methodology. Therefore the strategies of Unisul Virtual are focused on permanent activities to improve the tutor skills by training, support and pedagogical maintenance. The paper fi-nishes with results and proposals of perspectives and new lines of research.
\end{abstract}

\section{INTRODUCCIÓN: LA PROFESIONALIZACIÓN DE LOS ESTUDIOS UNIVERSITARIOS}

La idea de la profesionalización de los estudios no es ciertamente nueva. En los años sesenta en gran parte de Europa, una vez que se había conseguido la masificación de los estudios de enseñanza secundaria, se dió un gran impulso a los estudios de este nivel de carácter profesional para que tuvieran igual consideración académica y reconocimiento que los estudios de carácter teórico. Se trataba de 
ofrecer una educación que no estuviera tan marcada por el carácter academicista de los estudios sino que mejorara la futura inserción laboral de los estudiantes.

A partir de los años noventa la educación se desarrolla en un nuevo marco internacional, en el que destaca la caída del Muro de Berlín y la firma del Tratado de Maastricht. La preparación de los ciudadanos para un mundo globalizado planteaba nuevos retos a la educación. En particular, se hablaba de la necesidad de proporcionar a los ciudadanos una formación que les permitiera garantizar un crecimiento sostenible de las economías basadas en el conocimiento, las cuales dependen cada vez más del sector servicios. Había que conseguir la masificación de los estudios universitarios y, al mismo tiempo, que éstos garantizaran una mejor inserción laboral de los egresados universitarios.

Ante estos nuevos retos de la sociedad de la información, la educación ha sido sometida a un profundo proceso de revisión, especialmente en el ámbito universitario. Fruto de esta revisión se introduce en los vigentes Planes de Estudio universitarios, en la Titulación de Pedagogía, la asignatura del Practicum, en calidad de asignatura troncal tanto en el primer como en el segundo ciclos. De acuerdo a los descriptores, se define el Practicum como un "conjunto integrado de prácticas educativas que pongan en contacto al alumno con la realidad escolar" y "como conjunto integrado de prácticas tendentes a proporcionar experiencia directa sobre administración, planificación y dirección de instituciones y sistemas educativos". En definitiva, se trata de un tipo de asignatura de carácter profesionalizante que en el marco del Espacio Europeo de Educación Superior, que entrará en vigor a partir del año 2007, su papel se reforzará.

En el caso de la UNED se introduce en los Planes de Estudio como una asignatura troncal en dos niveles: Practicum I (10 créditos equivalentes a 100 horas lectivas) en el tercer curso. Los créditos del Practicum I se distribuyen de la siguiente manera: 70 horas de realización de las prácticas en la Institución correspondiente; 6 horas de asistencia a seminarios obligatorios en el Centro asociado y, 24 horas de tutoría. Los créditos del Practicum II.

El diseño de la asignatura del Practicum de Pedagogía de la UNED se ha realizado en dos modalidades: el Practicum específico o concertado y el Practicum abierto. El Practicum específico es la modalidad en la que el alumno que acude al centro de trabajo ha establecido un Convenio con la UNED. Este alumno es supervisado por un profesional colaborador. El Practicum abierto es la modalidad en la que el alumno acude a un centro de trabajo a realizar sus prácticas y que no ha firmado convenio con la UNED. En el caso de otros Practicum de la Facultad de Educación, en particular, en el de Educación Social, los alumnos que tienen una amplia experiencia práctica en el ejercicio profesional se acogen a la modalidad del reconocimiento de experiencia práctica, en la que se les exime de la realización de las horas de prácticas.

Por último, cabe señalar los ámbitos en los que se puede desarrollar el Practicum, en el ámbito de la Pedagogía son fundamentalmente dos: a) Pedagogía 
Socio-profesional; b) Pedagogía de las Instituciones educativas: contexto educativo; contexto empresarial; contexto comunitario.

\section{INNOVACIONES QUE INTRODUCE EL PRACTICUM DE PEDAGOGÍA}

La novedad y juventud de este tipo de formación no está exenta de dificultades. El acercamiento de los estudiantes al mundo laboral como parte de sus estudios obliga a cambios de distinta índole: la necesidad de modificar la organización interna; las relaciones externas universidad- centros de trabajo; la participación de varios profesionales de diferente perfil profesional; las dificultades para la evaluación; y, los diferentes perfiles de alumnos.

\section{El Practicum: una asignatura teórico-práctica}

Hasta ahora la universidad se encargaba casi exclusivamente de las enseñanzas de carácter teórico que se impartían siguiendo el estilo de enseñanza-aprendizaje tradicional. Las prácticas eran optativas y no figuraban en los planes de estudio por lo que su realización dependía del interés y buena voluntad de determinados alumnos, profesores y departamentos. Todas las asignaturas que se impartían en la universidad tenían un carácter eminentemente teórico y partían de un enfoque de aprendizaje en el que el alumno debía asimilar de manera memorística unos contenidos que para después debían ser plasmados de acuerdo a una serie de preguntas más o menos abiertas o con más o menos opciones de respuesta en un examen.

En la asignatura del Practicum la epistemología de la materia obliga a desarrollar un proceso inverso. El alumno combina las horas de prácticas con un trabajo de documentación, búsqueda bibliográfica e interpretación de la realidad donde interactúa. Esto supone que el alumno realice un aprendizaje muy personal en el que intervienen múltiples agentes profesionales. Asimismo el aula deja de ser el único espacio de aprendizaje ya que el contraste con la práctica se hace desde el centro de formación in situ. Esto ofrece muchas ventajas como contrastar fuentes de información, analizar casos, realizar observaciones directas de la práctica educativa, comparar experiencias de profesionales, etc. Así, la significación de los saberes se consigue en la realidad de los centros. En síntesis esta asignatura tiene un carácter epistemológico que se define por su carácter teórico-práctico.

\section{Las competencias de los docentes y discentes}

Hasta este momento la universidad se ha caracterizado por transmitir conceptos y teorías que debían memorizarse y aprehenderse, y que en un segundo momento se recogían en una prueba escrita. En el Practicum la palabra del profesor y el texto escrito dejan de ser los referentes básicos del aprendizaje, ya que la formación teóricopráctica no puede guiarse de la misma manera que la teórica. En el Practicum al alumno se le ofrecen una serie de herramientas que ayuden a buscar y a decidir cuáles 
son los materiales que van a permitir enriquecer su experiencia práctica. Se trata de que el alumno se convierta en el centro de este aprendizaje y que sea capaz de desarrollar o revisar algunas destrezas necesarias para la actualización y mejora de su práctica profesional. Desde esta perspectiva, la asignatura del Practicum permite al alumno el desarrollo de capacidades, habilidades y destrezas adquiridas en la Facultad, con el fin de que los alumnos logren mejores y más ajustados niveles de cualificación teórica, técnica y profesional. En este sentido se inicia o amplia el conocimiento de la realidad social e institucional que configura su profesión: personas, servicios, programas, ámbitos de intervención, problemas, etc., de manera directa y participante se fomenta la libertad y autonomía de los estudiantes, de su creatividad, iniciativa y responsabilidad en un contexto experiencial para el que deben asumir compromisos de acción y una progresiva participación en su dinámica: toma de decisiones, utilización de técnicas y recursos, valoraciones, etc.

Los profesores deben de convertirse en guías o fuentes de conocimiento. Su importancia es esencial, puesto que el profesorado no sólo organiza el contenido, las estrategias de aprendizaje y los métodos de evaluación, sino que además crea el clima y puede actuar como modelo empleando estrategias orientadas al Aprendizaje Permanente. Los profesores deben adquirir las siguientes competencias:

- el dominio de procesos que determinan la generación, apropiación y uso del conocimiento;

- la capacidad de incorporar las nuevas tecnologías de información y comunicación al aprendizaje;

- los deseos de propiciar y facilitar el aprendizaje;

- la capacidad para despertar el interés, la motivación y el gusto por aprender;

- la disponibilidad para aprender por cuenta propia y a través de la interacción con otros;

- las habilidades para estimular la curiosidad, la creatividad y el análisis;

- las aptitudes para fomentar la comunicación interpersonal y el trabajo en equipo;

- la imaginación para identificar y aprovechar oportunidades diversas de aprendizajey,

- la autoridad moral.

\section{Innovaciones de carácter metodológico}

El Practicum es una asignatura que se ajusta muy bien a los patrones metodológicos que se pretenden introducir en la universidad a través de la construcción del Espacio Europeo de Educación Superior que se articula alrededor de la noción de crédito. La introducción de los créditos en la nueva arquitectura supone que el examen no constituye el culmen del aprendizaje de una asignatura universitaria. El crédito distorsiona esta visión unilateral ya que se centra en el trabajo del estudiante que se le exige para alcanzar los objetivos de un programa, donde se especifican los objetivos en términos de resultados de aprendizaje y las competencias que deben adquirirse. El trabajo del estudiante, medido en ECTS, se refiere al tiempo exigido para completar todas 
las actividades planeadas como la asistencia a clases, seminarios, estudios privados, preparación de proyectos, exámenes, etc. Los créditos se asignan a todos los elementos del programa de estudios (tales como módulos, cursos, prácticas, trabajo de disertación, etc.) y reflejan la cantidad de trabajo que hay que realizar en contraste con la cantidad de trabajo necesario para completar un curso íntegro de estudio.

En el documento elaborado por la Oficina Europea de la Asociación Internacional de Educadores sociales (AIEJ I) se señala la importancia de desarrollar competencias teóricas y metodológicas. De entre todas podemos seleccionar las más relevantes respecto al Practicum I, como son:

- estar familiarizado con las teorías educativas básicas más reconocidas y aceptadas;

- tener capacidad para buscar y adquirir teorías y métodos educativos, psicológicos, sociológicos, antropológicos, etc. relevantes y adecuados e incluirlos en su trabajo;

- ser capaz de razonar las acciones y actividades basándose en teorías y métodos reconocidos;

- tener capacidad para contribuir en la generación de conocimientos, basándose en descripciones y reflexiones sobre la experiencia del trabajo socioeducativo.

A esto se añaden las competencias conductuales que se refieren a:

- dominar la terminología y los conceptos de la profesión y tomar parte en la creación de esta terminología;

- tener capacidad de interiorizar la ética y la moral de la profesión;

- tener capacidad de participar en debates, propuestas, borradores y acuerdos sobre la educación social;

- tener conocimiento de la terminología, conceptos y cimientos éticos de profesiones similares.

\section{LAS PECULIARES CARACTERÍSTICAS ORGANIZATIVAS DEL PRACTICUM EN LA UNED}

Las dificultades de organización del Practicum en una universidad a distancia han desembocado en la elaboración de unas Directrices específicas de Practicum de las titulaciones de la Facultad de Educación de la UNED que han sido aprobadas el 22 de marzo de 2006. A partir de este documento se establece el equipo de administración encargado de gestionar el Practicum. La Unidad de Gestión Académica de Practicum se mantiene con los siguientes miembros: Vicedecano de Practicum; Vicedecano de Ordenación Académica; Directores de departamentos; Coordinadores de las asignaturas de cada titulación.; un representante de la Facultad de Psicología; y, el Secretario de la Facultad (con voz pero sin voto).

La UNED es un modelo de universidad que responde al b-learning, en el que se combina perfectamente la docencia a distancia y la presencial. El Practicum se apoya 
en estos dos tipos de docencia por lo que están implicados distintos agentes. La organización de la docencia recae en los siguientes miembros: equipo docente de la Sede Central, el coordinador de cada asignatura; profesor tutor; y el profesional colaborador. El equipo docente se encarga de las funciones relativas a la docencia. El coordinador de cada asignatura de Practicum, elegido por los propios profesores entre los miembros del equipo docente, ejerce de coordinador del mismo. El profesor tutor ejerce su función docente en el Centro Asociado de acuerdo con la normativa vigente. El profesional colaborador es la persona con la que el estudiante realizará las prácticas en la entidad colaboradora.

Esta organización, algo más compleja que las asignaturas de carácter teórico, pretende responder a las necesidades de un alumnado heterogéneo que tiene que enfrentarse a múltiples dificultades: ubicación de los alumnos (territorio nacional, en el extranjero, en centros penitenciarios), heterogeneidad de las circunstancias personales (alumnos que trabajan, enfermedad embarazo, responsabilidades familiares). Los alumnos deben compaginar sus horarios con las horas de prácticas obligatorias.

\section{ORGANIZACIÓN DEL PRACTICUM DE LA FACULTAD DE PEDAGOGÍA}

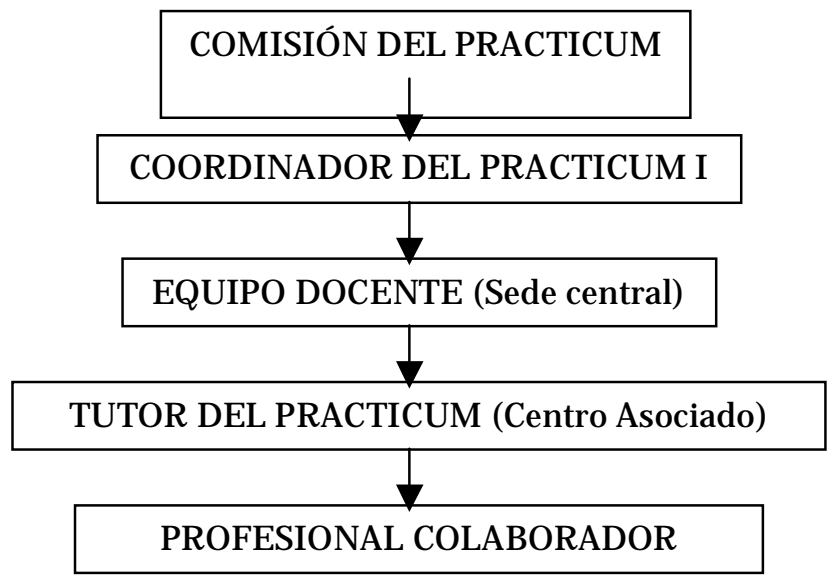

La UNED en calidad de universidad a distancia, dispone de diversos medios para impulsar el aprendizaje de los alumnos y, pone a disposición de los mismos una gran diversidad de recursos. Estos recursos permiten una impartición más homogénea de los contenidos del Practicum en los diversos Centros Asociados de la UNED. Entre estos cabe destacar:

- guía del curso en permanente actualización, que se publica en la UNED y que recoge las directrices y aspectos organizativos del Practicum;

- libros elaborados por el profesorado que amplían las directrices y permiten una mayor comprensión de los aspectos esenciales de la materia;

- tutorías que constituyen un lugar de encuentro de alumnos y profesores; 
- seminarios permanentes que constituyen un lugar de encuentro de todo tipo de profesionales implicados en el Practicum;

- convivencias que permiten a alumnos y profesores de la Sede Central trabajar los aspectos que no han sido comprendidos adecuadamente;

- videoconferencias constituyen un medio que pone en contacto a los alumnos de diversos Centros Asociados;

- TeleUned: permite colgar materiales visuales elaborados por el profesor de la Sede Central y que pueden ser consultados por el alumno en cualquier momento a través de un ordenador;

- RadioUNED: ofrece a los alumnos programas en los que se explican temas de actualidad relacionados con la asignatura;

- Además de estos recursos, la UNED dispone de todo tipo de documentos de carácter digital para el alumno como son LiberUNED que ofrece un catálogo amplio delibros electrónicos, etc.

\section{LOS FINES Y CONTENIDOS DEL PRACTICUM I DE PEDAGOGÍA DE LA UNED}

\section{Los fines del Practicum}

Los fines de la asignatura del Practicum I de Pedagogía que giran en torno a la destreza clave o nuclear de la observación se sintetizan en los siguientes:

- el conocimiento, profundidad y la reflexión de un ámbito de la intervención;

- la vinculación de la práctica con la teoría;

- la reflexión sobre los aspectos personales (autoevaluación) y sobre los aspectos profesionales durante el período de prácticas (centro, programas, profesionales y usuarios) (Gavari, 2006).

A partir de estos fines se establecen de forma general los objetivos generales, específicos y operativos y los productos, tal y como se recoge en el siguiente esquema:

\begin{tabular}{|c|c|c|}
\hline $\begin{array}{l}\text { FIN DEL PRACTICUM } \\
\text { - Conocimiento en profundidad } \\
\text { de un ámbito de la intervención. }\end{array}$ & $\begin{array}{l}\text { FIN DEL PRACTICUM } \\
\text { - Vinculación de la práctica oon } \\
\text { la teoría. }\end{array}$ & $\begin{array}{l}\quad \text { FIN DELPRACTICUM } \\
\text { - Reflexión sobre aspectos per- } \\
\text { sonales (autoevaluación) y profe- } \\
\text { sionales (centro, programas, } \\
\text { profesionales y usuarios). }\end{array}$ \\
\hline \multicolumn{3}{|c|}{ OBJETIVOS DEL PLAN DE PRÁCTICAS } \\
\hline $\begin{array}{l}\text { OBJETIVOS GENERALES } \\
\text { - Conocer desde el punto de } \\
\text { vista teórico y práctico un ámbito } \\
\text { dela intervención. }\end{array}$ & $\begin{array}{l}\text { OBJETIVOS GENERALES } \\
\text { - Ejercitarse en la planificación, } \\
\text { técnicas de la observación y del } \\
\text { diario para vincular teoría y } \\
\text { práctica. }\end{array}$ & $\begin{array}{l}\text { OBJETIVOS GENERALES } \\
\text { - Mejorar el autoconocimiento } \\
\text { (competencias, intereses, expec- } \\
\text { tativas, aptitudes, actitudes en } \\
\text { un ámbito profesional; }\end{array}$ \\
\hline
\end{tabular}




\begin{tabular}{|c|c|c|}
\hline & & $\begin{array}{l}\text { - mejora del conocimiento de } \\
\text { todos los elementos que inter- } \\
\text { vienen en el centro de prácticas; } \\
\text { - mejora de la comprensión y } \\
\text { análisis de las estrategias de } \\
\text { intervención entre educador y } \\
\text { educando. }\end{array}$ \\
\hline \multicolumn{3}{|c|}{ FASES DEL PLAN DE PRÁCTICAS } \\
\hline $\begin{array}{l}\text { OBJETIVOS ESPECÍFICOS } \\
\text { - Saber localizar y utilizar las } \\
\text { diversas fuentes documentales } \\
\text { propias de su ámbito de inter- } \\
\text { vención; } \\
\text { - justificar desde el punto de } \\
\text { vista teórico los principios teóri- } \\
\text { os que argumentan la interven- } \\
\text { ción en el centro; } \\
\text { - justificar desde un punto de } \\
\text { vista científico la intervención en } \\
\text { el centro. }\end{array}$ & $\begin{array}{l}\text { OBJETIVOS ESPECÍFICOS } \\
\text { - Conocer los problemas y deci- } \\
\text { siones implicados en la toma de } \\
\text { decisiones sobre el uso de la } \\
\text { metodología observacional; } \\
\text { - diseñar cómo se recoge la in- } \\
\text { formación a través de la observa- } \\
\text { ción; } \\
\text { - realizar un contraste entre lo } \\
\text { planificado y lo aprendido; } \\
\text { - realizar el análisis de conteni- } \\
\text { do del diario; } \\
\text { - recoger todo lo aprendido en } \\
\text { un informe. }\end{array}$ & $\begin{array}{l}\text { OBJETIVOS ESPECÍFICOS } \\
\text { - Identificar las funciones y } \\
\text { tareas propias del profesional de } \\
\text { la educación; } \\
\text { - fomentar el desarrollo de la } \\
\text { comprensión de todo lo que } \\
\text { sucede en el centro de prácticas } \\
\text { en su complejidad; } \\
\text { - conocer progresivamente la } \\
\text { dinámica del centro, la organiza- } \\
\text { ción y su funcionamiento; } \\
\text { - reflexionar sobre las estrate- } \\
\text { gias en la intervención educativa. }\end{array}$ \\
\hline $\begin{array}{l}\text { ACTIVIDADESY PRODUC- } \\
\text { TOS } \\
\text { - } \text { Revisar documentación (ex- } \\
\text { terna einterna); } \\
\text { - realizar entrevistas; } \\
\text { - trabajo personal en casa, acti- } \\
\text { vidades en el centro de prácticas, } \\
\text { actividades coordinadas on los } \\
\text { tutores de la universidad. }\end{array}$ & $\begin{array}{l}\text { ACTIVIDADES Y PRODUC- } \\
\text { TOS } \\
\text { - Elaborar un Plan de Prácticas, } \\
\text { el diario y el informe; } \\
\text { mecanismos de recogida de la } \\
\text { información a través de la ob- } \\
\text { servación; } \\
\text { - trabajo personal en casa, acti- } \\
\text { vidades en el centro de prácti- } \\
\text { cas, actividades coordinadas } \\
\text { con los tutores de la universi- } \\
\text { dad. }\end{array}$ & $\begin{array}{l}\text { ACTIVIDADES Y PRODUC- } \\
\text { TOS } \\
\text { - Analizar y evaluar toda la } \\
\text { información; } \\
\text { - presentar propuestas de me- } \\
\text { jora; } \\
\text { - trabajo personal en casa, acti- } \\
\text { vidades en el œntro de prácticas, } \\
\text { actividades coordinadas con los } \\
\text { tutores de la universidad. }\end{array}$ \\
\hline
\end{tabular}

Fuente: (Gavari, 2006)

\section{Los contenidos del Practicum}

Una delas dificultades que el alumno debe superar es la falta de un material escrito que el alumno debe memorizar sino que al alumno de le pide la entrega de unos documentos finales. Para la realización de esos documentos el alumno debe realizar las siguientes tareas:

- revisar documentación externa e interna en el centro; 
- realizar entrevistas;

- trabajo personal en casa, actividades en el centro de prácticas, actividades coordinadas con los tutores de la universidad;

- elaborar un Plan de prácticas, el diario y el informe;

- diseñar mecanismos de recogida de la información a través de la observación;

- analizar y evaluar toda la información recogida;

- presentar propuestas de mejora.

Esto supone una importante labor de búsqueda bibliográfica. El alumno obtiene directamente información de partida en conversaciones con personas de experiencia o conocimientos especiales sobre la realidad práctica en la que trabaja. Adquirir información por esta vía es relevante, y como señalan algunos autores: "este medio de obtener información, insuficientemente valorado, no debe descuidarse, pues existe información, y sobre todo experiencias, que se quedan sin transmitir por escrito" (Sierra Bravo, 1999:149). La obtención de información también se puede realizar de manera indirecta, lo que implica una labor previa de documentación o de investigación sobre las fuentes que pueden contener datos de interés sobre aspectos claves del Practicum.

A modo de esquema se recogen diversos tipos de documentos que el alumno puede considerar.

\begin{tabular}{|c|c|}
\hline Observación & Documentación y búsqueda bibliográfica \\
\hline $\begin{array}{l}\text { - Autobservación ("autodocumentación" que ya } \\
\text { posee el alumno). } \\
\text { - Heterobservación: búsqueda de documentos } \\
\text { de un área específica sobre cómo debenáa funcio- } \\
\text { nar un centro de esas características y sobre cómo } \\
\text { se produce el proceso educativo (niveles macro y } \\
\text { micro). }\end{array}$ & $\begin{array}{l}\text { - } \quad \text { Conocimientos y documentos propios. } \\
\text { - Materiales que ya se han trabajado en la universidad (las } \\
\text { propiasasignaturas, entreotros). } \\
\text { - } \quad \text { Documentación externa al centro que se refiere específica- } \\
\text { mente al área de intervención (centros específicos sobre el tema, } \\
\text { bibliotecas). } \\
\text { - Documentación interna al centro (guías de buenas prácticas, } \\
\text { programas, expedientes. } \\
\text { - Entrevistas con profesionales para subsanar la falta de docu- } \\
\text { mentación. }\end{array}$ \\
\hline
\end{tabular}

Fuente: Gavari

\section{Evaluación del alumno}

La evaluación del alumno es muy diferente del resto de las asignaturas de carácter teórico. Independientemente de la modalidad que se elija, la evaluación trata de valorar las competencias socioprofesionales desarrolladas por el estudiante de practicum, tanto de carácter transversal (trabajo en equipos profesionales, búsqueda de recursos, etc.), como de carácter específico (observación participante, análisis de elementos relevantes del diario, etc.).

La metodología de evaluación del practicum se basa en el concepto de portfolio que permite tener en cuenta tanto los procesos como resultados reflejados por el estudiante y los otros agentes implicados en su practicum en los distintos elemen- 
tos/componentes: Plan de Prácticas, informe, informe del tutor, informe del profesional colaborador (practicum concertado), prueba presencial, etc. (Figura 1)

\section{Figura 1. Elementos de la evaluación}

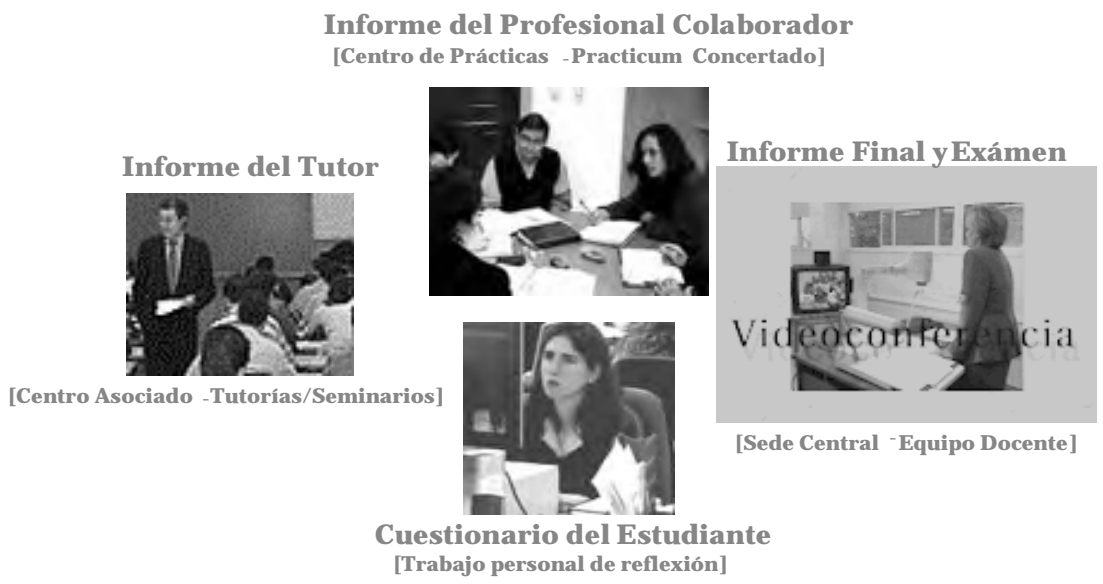

Cada uno de estos elementos son necesarios para proceder a la evaluación del Practicum. Se presenta una tabla resumen de estos elementos indicando dónde se hace el envío en función de que se presenten en junio o septiembre. (Tabla 1)

Tabla 1. Tabla resumen de verificación de elementos de la evaluación del practicum

\begin{tabular}{|c|c|c|}
\hline \multicolumn{2}{|c|}{ Modalidades } & \\
\hline Concertado & Abierto & Elementos/Componentes de la Evaluación del Practicum \\
\hline 邓 & Х & Ficha del Estudiante. \\
\hline 凶 & 凶 & Plan de Prácticas \\
\hline 凶 & 凶 & Informe del Tutor \\
\hline 区 & $\square$ & Informe del Profesional Colaborador \\
\hline $\bar{Q}$ & $\bar{\Delta}$ & Cuestionario de evaluación del estudiante \\
\hline $\bar{\Delta}$ & 区 & Informe \\
\hline 区 & Х & Examen/Prueba presencial \\
\hline
\end{tabular}

La Ficha del estudiante se envía al profesor de la sede central una vez realizada autorizado el inicio del practicum por el tutor indicando la modalidad correspondiente.

1. El Plan de Prácticas recoge las actividades, calendario y plan de trabajo establecido conjuntamente por el tutor y profesional colaborador. Se remite junto con el informe con firma del tutory sello del centro asociado.

2. El Informe del Tutor recoge la valoración individualizada de los estudiantes en función de la modalidad de practicum. Se remite en junio, aunque el estudiante no haya entregado el informe. 
3. El Informe del Profesional colaborador valora las actividades desarrolladas por el alumno, el grado de aprovechamiento y su nivel de participación. Se remite en sobre cerrado [Confidencial] junto con el informe.

4. El cuestionario de evaluación del estudiante recoge las valoraciones realizadas por el estudiante del desarrollo del practicum y de los agentes implicados en el mismo.

5. El informe presenta unas orientaciones específicas para cada modalidad del practicum.

6. El examen o prueba presencial permite verificar aspectos concretos en relación con las prácticas realizadas por lo que se recomienda asistir al examen una vez finalizada la elaboración del portfolio. Para la realización del mismo no se permite consultar la guía ni ningún otro material.

Algunos de los criterios de evaluación del practicum son los siguientes:

- Presentar en tiempo y forma los diferentes componentes del practicum de cada modalidad.

- Respetar los procedimientos de asignación de centro de prácticas y seguimiento validado por el tutor.

- Tener validado el Plan de Prácticas para el inicio delas prácticas.

- Participar en los seminarios obligatorios programados en el centro asociado o a través de fórmulas alternativas consensuadas con el tutor que posibiliten el logro de los objetivos de esta materia, teniendo en cuenta las posibilidades tecnológicas de apoyo que brinda la metodología a distancia propia de esta Universidad.

- Tener una calificación de apto en todos los componentes del portfolio para proceder a su ponderación global.

- Los estudiantes residentes en el extranjero o pertenecientes al programa de centros penitenciarios, las funciones de tutor se asumen por el profesor asignado del equipo docente [ver relación de profesores].

Los plazos y fechas clave de entrega del informe son dos, se podría añadir un tercero si se cumplen los requisitos establecidos por la UNED:

- Convocatoria de Junio: Se entrega en el Centro Asociado en la fecha fijada por el Tutor.

- Convocatoria de Septiembre: Se envía a la Sede Central:

UNED Secretaría de Educación

Practicum II de Educación Social. Centro Asociado de:

Paseo Senda del Rey, 7. Despacho 5 (Planta menos 2)

28040 Madrid.

- Exámenes extraordinarios de Diciembre [Consultar Guía de la Carrera]. 
En el informe de evaluación del alumno, el Profesor Tutor se refiere a aspectos tales como: asistencia y participación en los seminarios presenciales; visita a las instituciones colaboradoras; entrevistas personales de carácter voluntario; valoración general del trabajo realizado para la redacción de la Memoria.

El cuestionario de evaluación del Colaborador profesional recoge información sobre los siguientes aspectos: asistencia y puntualidad; ajuste al plan de prácticas aprobado; espíritu de colaboración con el centro; capacidad para desarrollar un trabajo en equipo; discreción sobre los datos y, calidad del trabajo práctico.

\section{ALGUNAS RECOMENDACIONES PARA EL ALUMNO RESPECTO AL CENTRO DE PRÁCTICAS}

Una vez que el alumno sabe en qué centro va a realizar sus prácticas debe ser consciente de algunas cuestiones para no perjudicar las relaciones establecidas entre alumnos, profesores y los profesionales. Lo más importante es reconocer la generosidad y la oportunidad que el centro de prácticas brinda al alumno y la posibilidad de realizar sus prácticas. El alumno por su parte debe ser consciente de que en todo momento es un invitado y que él debe ser quien se adapte a la institución y no al revés. La actuación del alumno es muy importante ya que de él dependerá la posibilidad de que en un futuro próximo acudieran al centro otros alumnos en prácticas o incluso que posteriormente el centro le contratara como profesional. El alumno en prácticas debe evitar a toda costa cualquier tipo de conflicto o enfrentamiento entre los profesionales. Si por cualquier motivo el alumno decidiera abandonar el centro de prácticas debería siempre agradecer al centro la oportunidad que le ha brindado. Algunos comportamientos que debería adoptar son:

- conocer las normas del centro adoptando una actitud de respeto y aceptación de las mismas;

- respetar los horarios y ajustarse al ritmo del trabajo;

- mantener el orden en los espacios propios y comunes;

- participar activamente en cada una de las tareas que se encomiendan al alumno, aunque no sean tareas de observación;

- mostrar una actitud activa e iniciativa propia a la hora de emprender actividades o tareas sin que sea necesaria la continua supervisión o propuesta de soluciones por parte del supervisor de las prácticas;

- no desautorizar órdenes de otros profesionales;

- no criticar opiniones o consejos de otros profesionales aunque no coincidan con los propios;

- garantizar la confidencialidad de la información que se ha adquirido;

- manejar con discreción la información que se haya obtenido dentro del centro de prácticas;

- a la hora de pedir información o de entrevistar a los profesionales se debe actuar con tacto e intuición acerca de las cuestiones más conflictivas;

- tener presente que no es necesario conocerlo todo sino lo más relevante; 
- si por algún motivo los profesionales que trabajan en el centro no quieren aportar información no preocuparse ni tomar una actitud negativa, como alternativa se puede entrevistar a los profesionales para conseguir la información que se necesita (Gavari, 2006).

\section{CONCLUSIONES}

El Practicum está suponiendo un gran reto al que dar respuesta en todas las universidades españolas; y, en particular, a la UNED por ser esta una universidad a distancia. Desde la puesta en marcha de esta asignatura y gracias al esfuerzo de profesores y personal de administración de la Sede Central y de los Centros Asociados, hasta la actualidad se han dado importantes avances y la asignatura, día a día, tiene unos fines más claros. La Unidad de gestión que se ha creado puede servir como modelo a otras universidades a distancia. No olvidemos el importante papel de los miembros de la administración en universidades tan numerosas. Una vez solucionados los problemas de gestión del Practicum, podemos aprovechar la oportunidad única de una asignatura novedosa y tan innovadora en la que los profesores universitarios claramente se posicionan como guías del aprendizaje y los alumnos se convierten en motor del aprendizaje que ellos mismos construyen. Esto permite a los alumnos que vayan adquiriendo destrezas para el aprendizaje permanente que debe quedar como una actitud en esta sociedad actual de la información, en la que la valoración de los sucesos y de la información que nos rodea es fundamental. Si se adquieren unas buenas actitudes de base, los alumnos se acostumbrarán a emitir juicios de valor y a concluir sobre bases científicas y no meramente intuitivas. 


\section{REFERENCIAS BIBLIOGRÁFICAS}

AA.VV (2005): Guía del Practicum II. Madrid, UNED.

AA.VV (1988): La educación postsecundaria ante la sociedad del conocimiento y de las comunicaciones. III Semana Monográfica. Madrid, Fundación Santillana.

Coll, C. (2002): Observación y análisis de las prácticas de educación escolar. Barcelona, UOC.

D’Hainaut, L. (1985): Objetivos didáctioos y programación. Barcelona, Oikos.tan.

Espinosa Vergara, M. (1987): Evaluación de Proyectos Sociales. Buenos Aires, Humanitas.

Eurydice (2000): Lifelong Learning: the Contribution of Education Systems in the EU. Brussels, European Union.

Eurydice (2003): Las competencies Clave. Brussels, European Union.

Evertson, J .M. y Green, J . (1998): “La observación como indagación y método" en M.C. Wittrock (ed): la investigación de la enseñanza, II. Métodos cualitativos y de observación. Barcelona: Paidós.

Gagné, R. (1983): La planificación de la enseñanza. Trillas: México.

García Aretio, L. (1997): Unidades Didácticas y Guías Didácticas. Madrid: UNED.

Gavari, E. (2005): La educación en Francia y en Italia en la segunda mitad del siglo XX. Madrid: UNED.

Gavari, E. (2006): Estrategias para la observación de la práctica educativa. Barcelona: Editorial Ramón Areces.

Julia I Bosch, A. (1995): El educador social: una figura profesional surgida de diversas prácticas profesionales en "I Congreso Estatal del Educador Social. Presente y futuro en la Educación Social. Barcelona: BIBLÀRIA.

López- Barajas, E. (1996): Las historias de vida y la investigación biográfica. Fun- damentos y metodología. Madrid: UNED.

Mager, R. (1973): Formulación operativa de objetivos didácticos. Madrid: Marova.

Martínez, M. y Esteban, F. (2005): “Una propuesta de formación ciudadana para el EEES" en Revista española de pedagogía, nº 230, año LXIII, enero abril 2005.

Martínez, C. (Coord.) (2004): Técnicas e instrumentos de recogida y análisis de datos. UNED: Madrid.

Martínez, E. (1999): Planificación, programación y evaluación dela aoción educativa. Málaga: FACEP.

Medina, A. (1996): “La autobiografía, opción de formación del profesorado. Limitaciones y posibilidades" en Las Historias de vida y la investigación biográfica. Fundamentos y metodología. UNED: Madrid.

Medina, R. (2005): “Misiones y funciones de la universidad en el Espacio Europeo de Educación Superior" en Revista Española de Pedagogía, no 230, año LXIII, enero-abril 2005.

Merino Fernández, J.V. (2002): “La planificación de la acción socio-cultural" en Programas de Animación sociocultural. Madrid: UNED.

Pain, A. (1990): Cómo realizar un proyecto de capacitación. Granica: Argentina.

Postic, M. Y De Ketele, J.M. (1988): Observar las situaciones educativas. Madrid: Narcea.

Postic, M (1996): Observación y formación de profesores. Madrid: Ediciones Morata.

Pérez Serrano, G. (1993): Elaboración de Proyectos Sociales. Casos Prácticos. Madrid: Narcea.

Petersen, W.H. (1967): El currículo en la Escuela Primaria. El Ateneo: Buenos Aires. 
Pujadas Muñoz, J J : (1992): El método biográfico: el uso delas historias devida en Ciencias Sociales. Madrid: CIS.

Rotger, B. (1985): El proceso programador en la escuela. Madrid: Escuela Española. diario la actividad personal. Madrid: Editorial Escuela Española.

Rye, R. y Torbjorrnsen, A. (2004): Competente-based curricula-The Norwegian [en línea]. Disponible en www.cidree.org.

Rotger, B. (1980): Cómo elaborar un Proyecto de trabajo práctico y preparar a

\title{
PALABRAS CLAVE
}

Formación práctica, observación, personal docente.

\section{KEY WORDS}

Practical formation, observation, educational personnel.

\section{PERFIL ACADÉMICO DE LA AUTORA}

Elisa Gavari Starkie, Doctora en Filosofía y Ciencias de la Educación es, desde el año 2001, profesora adscrita al Departamento de Historia de la Educación y Educación Comparada en la UNED. Imparte docencia en la Licenciatura de Pedagogía y en la Diplomatura de Educación Social así como en cursos del Programa de Formación del Profesorado. Ha desarrollado su actividad investigadora en torno a las políticas educativas europeas.

Dirección postal:

\author{
Facultad de Educación \\ Paseo Senda del Rey 7 \\ UNED \\ 28040 Madrid, España. \\ E-mail: egavari@edu.uned.es
}

Fecha recepción del artículo: 07. 03. 2006

Fecha aceptación del artículo: 17. 03. 2006 